ments, we may proceed afresh, since $D_{p}$ is of the same form as $D$, but contains only the functions $f_{2}, f_{3}, \cdots, f_{p}$. Reasoning as above, we find that these $p-1$ functions are linearly dependent, and hence also the $p$ given functions, unless the $(p-2)$-rowed determinant in the upper right-hand corner of $D_{p}$ vanishes identically. In the latter case we must continue in the same way, until we finally reach a determinant in the upper right-hand corner of $D$ which is not identically zero. But there must be one such, unless $f_{p}(t)$ is itself identically zero, in which case the given $p$ functions are linearly dependent. The theorem for analytic functions as stated in Mr. Morse's note therefore follows.

It may be of interest to point out that many-if not allof the theorems on linear dependence in which wronskians or determinants and matrices constructed like wronskians are involved* have their analogues in corresponding theorems in which appear determinants and matrices resembling the determinant $D$.

HARVARD UNIVERSITY, July, 1916.

\title{
TRANSLATION SURFACES ASSOCIATED WITH LINE CONGRUENCES.
}

\author{
BY PROFESSOR O. E. GIENN.
}

(Read before the American Mathematical Society, October 28, 1916.)

\section{§1. Introduction.}

IN a note published in the BuLLETIN in 1914 $\uparrow$ I established an algorism on a class of surfaces associated with line congruences in 3-space, which result by translation from invariants of plane $n$-lines. It is the purpose of this paper to apply symbolical methods to the study of some properties of these surfaces.

Two non-homogeneous forms of respective orders $m, n$, in Plücker's line coordinates $p_{1}, p_{2}, q_{1}, q_{2}, r[r=(p q)]$, considered together, represent a congruence $(m, n)$. For the sake of symmetry let the variables be changed to the homogeneous system

\footnotetext{
* Such, for instance, as are given by Bôcher, loc. cit., and by Curtiss, Math. Annalen, vol. 65 (1908), pp. 282-298.

$\dagger$ Bulletin, vol. 20 , p. 233.
} 


$$
\begin{aligned}
\xi_{1}=p_{1}, & \xi_{2}=p_{2}, \quad \xi_{3}=q_{1}, \quad \xi_{4}=q_{2}, \quad \xi_{5}=r, \\
\xi_{6}=1 ; & \left(\xi_{5}=\xi_{1} \xi_{4}-\xi_{2} \xi_{3}\right) .
\end{aligned}
$$

Then these forms may be written

$$
\begin{gathered}
f_{m}=\left(\alpha_{1} \xi_{1}+\cdots+\alpha_{6} \xi_{6}\right)^{m}=\alpha_{\xi}{ }^{m}=\alpha_{\xi}{ }^{m}=\cdots, \\
g_{n}=\left(\beta_{1} \xi_{1}+\cdots+\beta_{6} \xi_{6}\right)^{n}=\beta_{\xi}{ }^{n}=\beta_{\xi}{ }^{\prime n}=\cdots
\end{gathered}
$$

The generating line of $(m, n)$ is

$$
\xi_{6} x_{1}-\xi_{1} x_{3}-\xi_{3} x_{4}=0, \quad \xi_{6} x_{2}-\xi_{2} x_{3}-\xi_{4} x_{4}=0,
$$

and this generator is contained in an arbitrary plane $w_{x}=0$ provided

$$
\begin{aligned}
& \xi_{1} w_{1}+\xi_{2} w_{2}+\xi_{6} w_{3}=0, \\
& \xi_{3} w_{1}+\xi_{4} w_{2}+\xi_{6} w_{4}=0, \\
& \xi_{5} w_{1}+\xi_{4} w_{3}-\xi_{2} w_{4}=0 .
\end{aligned}
$$

\section{$\S 2$. The Surfaces Associated with $(m, 1)$.}

A canonical form of $(m, 1)$ is furnished by the pair

$$
f_{m}=0, \quad \xi_{5}+1=0,
$$

and if we replace $\xi_{5}$ by -1 in $f_{m}$ the latter becomes the nonhomogeneous

$$
\left(\alpha_{1} \xi_{1}+\cdots+\alpha_{4} \xi_{4}+\left(\alpha_{6}-\alpha_{5}\right)\right)^{m},
$$

or, setting $\alpha_{6}-\alpha_{5}=\alpha_{5}$ and introducing, for homogeneity, a new $\xi_{5}(=1)$ in place of $\xi_{6}, f_{m}$ becomes the quinary form

$$
f_{1 m}=\left(\alpha_{1} \xi_{1}+\cdots+\alpha_{5} \xi_{5}\right)^{m}=\alpha_{\xi}{ }^{m}=\alpha_{\xi}{ }^{m} .
$$

From (1) we obtain, after replacing the old $\xi_{5}$ by -1 , and solving,

$$
\xi_{i}=\lambda_{i} \xi_{1}+\mu_{i} \xi_{5} \quad(i=1, \cdots, 5),
$$

where $\xi_{5}=1$, and

$$
\begin{aligned}
\lambda_{1} & =\mu_{5}=1, \quad \mu_{1}=\lambda_{5}=0, \quad \lambda_{2}=-w_{1} / w_{2}, \\
\mu_{2} & =-w_{3} / w_{2}, \quad \lambda_{3}=w_{4} / w_{3}, \quad \mu_{3}=-w_{2} / w_{3}, \\
\lambda_{4} & =-w_{1} w_{4} / w_{2} w_{3}, \quad \mu_{4}=\left(w_{1} w_{2}-w_{3} w_{4}\right) / w_{2} w_{3} .
\end{aligned}
$$


We now eliminate $\xi_{2}, \xi_{3}, \xi_{4}$ from $f_{1 m}$ by the substitutions (2), giving

$$
\begin{aligned}
F\left(\xi_{1}, \xi_{5}\right)=\left(\alpha_{\lambda} \xi_{1}+\alpha_{\mu} \xi_{5}\right)^{m}=a_{\xi}{ }^{m}= & a_{{ }_{\xi}}{ }^{m} \\
& \left(\alpha_{\lambda}=\alpha_{1} \lambda_{1}+\cdots+\alpha_{5} \lambda_{5}\right) .
\end{aligned}
$$

The coefficients of $F$ are rational expressions in the planar coordinates $w_{1}, \cdots, w_{4}$; hence any invariant $I$ of this binary form translates into a homogeneous quantic $\varphi(w)$ in the $w$ 's representing the surface enveloped by the plane $w_{x}=0$ when the latter moves so as to intersect the congruence $(m, 1)$ in an $m$-line which maintains the property represented, projectively, by $I=0$. Since every invariant of $F$ is a linear combination of products of factors of type

$$
\left(a a^{\prime}\right)=a_{1} a_{2}{ }^{\prime}-a_{2} a_{1}{ }^{\prime} ; \quad I=\Sigma k\left(a a^{\prime}\right)^{p}\left(a a^{\prime \prime}\right)^{q} \cdots,
$$

we must compute $\left(a a^{\prime}\right)$ in order to obtain $\varphi(w)$ explicitly.

We find

$$
\begin{gathered}
\left(a a^{\prime}\right)=\alpha_{\lambda} \alpha_{\mu}{ }^{\prime}-\alpha_{\mu} \alpha_{\lambda}{ }^{\prime}=\sum_{i=1}^{4} \sum_{j=1}^{\prime 5-i}\left(\alpha \alpha^{\prime}\right)_{i i+j}(\lambda \mu)_{i i+j}, \\
\left(\alpha \alpha^{\prime}\right)_{p q}=\alpha_{p} \alpha_{q}{ }^{\prime}-\alpha_{q} \alpha_{p}{ }^{\prime} .
\end{gathered}
$$

Theorem. The class (order in $w_{1}, \cdots, w_{4}$ ) of the translation surface $\varphi(w)=0$ equals twice the weight of the invariant $I$.

In proof, it is evident from (4) that the order of $\varphi$ in $\lambda_{1}$, $\cdots, \lambda_{5}, \mu_{1}, \cdots, \mu_{5}$ is twice the weight of $I$; but these quantities $\lambda, \mu$, when reduced to the common denominator $w_{2} w_{3}$, have numerators of dimensions 2 in $w_{1}, \cdots, w_{4}$. Nevertheless, from (3), we calculate

$$
\begin{gathered}
(\lambda \mu)_{12}=-w_{3} / w_{2}, \quad(\lambda \mu)_{13}=-w_{2} / w_{3}, \\
(\lambda \mu)_{14}=\left(w_{1} w_{2}-w_{3} w_{4}\right) / w_{2} w_{3}, \quad(\lambda \mu)_{15}=1,
\end{gathered}
$$

$$
\begin{gathered}
(\lambda \mu)_{23}=\left(w_{1} w_{2}+w_{3} w_{4}\right) / w_{2} w_{3}, \quad(\lambda \mu)_{24}=-w_{1}^{2} / w_{2} w_{3}, \\
(\lambda \mu)_{25}=-w_{1} / w_{2}, \quad(\lambda \mu)_{34}=-w_{4}^{2} / w_{2} w_{3}, \\
(\lambda \mu)_{35}=w_{4} / w_{3}, \quad(\lambda \mu)_{45}=-w_{1} w_{4} / w_{2} w_{3} .
\end{gathered}
$$

Hence the theorem is proved.

The explicit symbolical form of $\varphi(w)$ is manifest from (4) and (5) in a form free from adventitious factors; * in fact $\left(a a^{\prime}\right)$ is

* If $I$ is the discriminant of $F$ the order of $\varphi(w)$ is $2 m(m-1)$. Nonsymbolical methods used in my former paper suggested $4 m(m-1)$ for this order, but the present theory shows that there is always an adventitious factor (a product of powers of $w_{1}, \cdots, w_{4}$ ) reducing it to $2 m(m-1)$. That no further reduction is possible is shown by an example in my previous paper. 
proportional to

$$
\begin{aligned}
W= & \left(\alpha \alpha^{\prime}\right)_{24} w_{1}^{2}+\left(\alpha \alpha^{\prime}\right)_{13} w_{2}^{2}+\left(\alpha \alpha^{\prime}\right)_{12} w_{3}^{2}+\left(\alpha \alpha^{\prime}\right)_{34} w_{4}^{2} \\
& +\left(\alpha \alpha^{\prime}\right)_{14}\left(w_{3} w_{4}-w_{1} w_{2}\right)-\left(\alpha \alpha^{\prime}\right)_{23}\left(w_{3} w_{4}+w_{1} w_{2}\right) \\
& +\left(\alpha \alpha^{\prime}\right)_{25} w_{1} w_{3}-\left(\alpha \alpha^{\prime}\right)_{15} w_{2} w_{3}+\left(\alpha \alpha^{\prime}\right)_{45} w_{1} w_{4} \\
& -\left(\alpha \alpha^{\prime}\right)_{35} w_{2} w_{4}
\end{aligned}
$$

and $\left(a a^{\prime \prime}\right)$ differs from $W$ only by the replacement of $\left(\alpha \alpha^{\prime}\right)$ by $\left(\alpha \alpha^{\prime \prime}\right)$.

The degree of $\varphi(w)$ in the coefficients of $f_{1 m}$ equals the number of symbols in $I$.

For illustration, if $m=2$ the only invariant of $F\left(\xi_{1}, \xi_{5}\right)$ is its discriminant $\left(a a^{\prime}\right)^{2}$. We may write the quinary form $f_{12}$ as follows:

$$
f_{12}=\Sigma a_{i j} \xi_{i} \xi_{j}=\alpha_{\xi}^{2} \quad\left(i, j=1, \cdots, 5 ; a_{i j}=a_{j i}\right) .
$$

Squaring $W$ we readily compute $\varphi(w)$, here a surface of class 4 and degree 2, viz.,

$$
\begin{aligned}
\varphi(w)=\frac{1}{2} W^{2}=\left(a_{22} a_{44}-a_{24}{ }^{2}\right) w_{1}{ }^{4} \\
+2\left(a_{14} a_{24}-a_{44} a_{12}+a_{23} a_{24}-a_{22} a_{34}\right) w_{1}{ }^{3} w_{2}+\cdots .
\end{aligned}
$$

$\S 3$. The Congruence $(m, n)$.

Assuming the linear form of the pair representing the congruence $(m, 1)$ in canonical form $\xi_{5}+1$ enabled us to derive for the result of the elimination (2) a binary form $F$ whose invariants represent projective properties of the $m$-line in which $w_{x}=0$ cuts $(m, 1)$. We now show that the corresponding elimination in the case of a plane cutting $(m, n)$ gives a pair of ternary forms whose simultaneous invariants represent projective properties of the $m n$-line of intersection.

Solution of equations (1) with $\xi_{5} \neq-1$, gives

$$
\xi_{i}=\lambda_{i} \xi_{2}+\mu_{i} \xi_{4}+\nu_{i} \xi_{6} \quad(i=1, \cdots, 6),
$$

where

$$
\begin{gathered}
\lambda_{1}=-w_{2} / w_{1}, \quad \lambda_{2}=1, \quad \lambda_{3}=0, \quad \lambda_{4}=0, \\
\lambda_{5}=w_{4} / w_{1}, \quad \lambda_{6}=0, \\
\mu_{1}=0, \quad \mu_{2}=0, \quad \mu_{3}=-w_{2} / w_{1}, \quad \mu_{4}=1, \\
\mu_{5}=-w_{3} / w_{1}, \quad \mu_{6}=0, \\
\nu_{1}=-w_{3} / w_{1}, \quad \begin{array}{l}
\nu_{2}=0, \quad \nu_{3}=-w_{4} / w_{1}, \quad \nu_{4}=0, \\
\nu_{5}=0, \quad \nu_{6}=1,
\end{array}
\end{gathered}
$$


and, employing (7) to eliminate $\xi_{1}, \xi_{3}, \xi_{5}$ from $f_{m}, g_{n}$, there result the respective ternary forms

$$
\begin{aligned}
& f_{1 m}=\left(\alpha_{\lambda} \xi_{2}+\alpha_{\mu} \xi_{4}+\alpha_{\nu} \xi_{6}\right)^{m}=a_{\xi}{ }^{m}={a_{\xi}}^{{ }^{m}}=\cdots, \\
& g_{1 n}=\left(\beta_{\lambda} \xi_{2}+\beta_{\mu} \xi_{4}+\beta_{\nu} \xi_{6}\right)^{n}=b_{\xi}{ }^{n}=b_{\xi}{ }^{\prime n}=\cdots,
\end{aligned}
$$

where, for example,

$$
\alpha_{\lambda}=\alpha_{1} \lambda_{1}+\alpha_{2} \lambda_{2}+\cdots+\alpha_{6} \lambda_{6} .
$$

Any invariant $I$ of the pair of forms $f_{1 m}, g_{1 n}$ is a linear combination of products of third order determinants of the two types

$$
\left(a a^{\prime} a^{\prime \prime}\right), \quad\left(a a^{\prime} b\right) .
$$

Evaluating the typical case of the second type, we deduce, by a known theorem on determinants of arrays,

$$
\begin{gathered}
\left(a a^{\prime} b\right)=\left|\begin{array}{ccc}
\alpha_{\lambda} & \alpha_{\mu} & \alpha_{\nu} \\
\alpha_{\lambda}{ }^{\prime} & \alpha_{\mu}{ }^{\prime} & \alpha_{\nu}{ }^{\prime} \\
\beta_{\lambda} & \beta_{\mu} & \beta_{\nu}
\end{array}\right|=\sum_{i, j, k}\left(\alpha \alpha^{\prime} \beta\right)_{i j k}(\lambda \mu \nu)_{i j k} \\
\left(i, j, k=1, \cdots, 6 ; i<j<k,\left(\alpha \alpha^{\prime} \beta\right)_{i j k}=\left|\alpha_{i} \alpha_{j}^{\prime} \beta_{k}\right|\right) .
\end{gathered}
$$

The number of terms in the summation in (8) is ${ }_{6} C_{3}=20$. Substitution from (8) etc. in

$$
I=\Sigma k\left(a a^{\prime} a^{\prime \prime}\right)^{p}\left(a a^{\prime} b\right)^{q} \ldots
$$

gives a homogeneous form $\varphi(w)$ of degree in the coefficients of $f_{1 m}, g_{1 n}$, jointly, equal to the number of symbols occurring in $I$.

We now prove that the class of this surface equals twice the number of determinant factors $\left(a a^{\prime} b\right), \cdots$ occurring in any term of $I$. This number equals the power of the determinant of the collineations in the invariant relation for $I, i$. e., the index. It is called the weight of $I$ by some writers, and, with this terminology, we now require the proof of the precise theorem stated in $\S 2$, for the present ternary case and congruence $(m, n)$.

Calculating the 20 determinants $(\lambda \mu \nu)_{i j k}$ and writing, for brevity, $(\lambda \mu \nu)_{i j k}=(i, j, k)$, we have

$$
\begin{array}{ll}
(1,2,3)=w_{2} w_{3} / w_{1}^{2}, & (1,2,4)=-w_{3} / w_{1}, \\
(1,2,5)=w_{3}^{2} / w_{1}^{2}, & (1,2,6)=0,
\end{array}
$$


(9)

$$
\begin{array}{ll}
(1,3,4)=-w_{2} w_{4} / w_{1}^{2}, & (1,3,5)=0 \\
(1,3,6)=w_{2}^{2} / w_{1}^{2}, & (1,4,5)=w_{3} w_{4} / w_{1}^{2} \\
(1,4,6)=-w_{2} / w_{1}, & (1,5,6)=w_{2} w_{3} / w_{1}^{2} \\
(2,3,4)=w_{4} / w_{1}, & (2,3,5)=-w_{3} w_{4} / w_{1}^{2} \\
(2,3,6)=-w_{2} / w_{1}, & (2,4,5)=0 \\
(2,4,6)=1, & (2,5,6)=-w_{3} / w_{1} \\
(3,4,5)=w_{4}^{2} / w_{1}^{2}, & (3,4,6)=0 \\
(3,5,6)=w_{2} w_{4} / w_{1}^{2}, & (4,5,6)=-w_{4} / w_{1}
\end{array}
$$

Since the non-vanishing fractions in $w_{1}, \cdots, w_{4}$ all have second order numerators and a common denominator $w_{1}^{2}$, the theorem is proved. Substitution of these results in (8) and the results from (8) in $I$ gives the explicit form of the translation surface $\varphi(w)$, in a form free from extraneous factors.

It is obvious that a complete set of invariants gives, in the present case of the congruence $(m, n)$ or in the previous binary case of $(m, 1)$, a fundamental system of translation surfaces. For the congruence $(2,2)$, cut by a plane in a quadrilateral, the complete system consists of four surfaces $\left(a a^{\prime} a^{\prime \prime}\right)^{2},\left(b b^{\prime} b^{\prime \prime}\right)^{2}$, $\left(a a^{\prime} b\right)^{2},\left(a b b^{\prime}\right)^{2}$, all of degree 3 and class 4 .

University of Pennsyluania.

\title{
PAPPUS. INTRODUCTORY PAPER.
}

\author{
BY DR. J. H. WEAVER.
}

(Read before the American Mathematical Society, April 24, 1915.)

ONE of the most wholesome tendencies in the study of mathematics today is the desire to give increased attention to the history and genesis of the subject. This tendency has led to a more careful study of the works of the old Greek mathematicians. Of these Pappus of Alexandria was among the last, and from the point of view of the historian one of the most important because it is in his works that we have the only authentic account of the lost works of a large number of preceding mathematicians. 